\title{
Polyp Localization and Segmentation in Colonoscopy Images by Means of a Model of Appearance for Polyps
}

\author{
Jorge Bernal \\ Computer Science Department \& Computer Vision Center, Edifici Q/O Campus UAB, Cerdanyola del Valles, Barcelona, Spain \\ Advisor/s: F. Javier Sánchez, Fernando Vilariño \\ Date and location of PhD thesis defense: 17 December 2012, Autonomous University of Barcelona
}

Received 24th Jan 2014; accepted 25th May 2014

\section{Summary of the thesis}

Colorectal cancer is the fourth most common cause of cancer death worldwide and its survival rate depends on the stage in which it is detected on hence the necessity for an early colon screening. There are several screening techniques but colonoscopy is still nowadays the gold standard, although it has some drawbacks such as the miss rate. Our contribution, in the field of intelligent systems for colonoscopy, aims at providing a polyp localization and a polyp segmentation system based on a model of appearance for polyps.

To develop both methods we define a model of appearance for polyps, which describes a polyp as enclosed by intensity valleys. The novelty of our contribution resides on the fact that we include in our model aspects of the image formation and we also consider the presence of other elements from the endoluminal scene such as specular highlights and blood vessels, which have an impact on the performance of our methods. In order to develop our polyp localization method we accumulate valley information in a smart way order to generate energy maps related to the likelihood of polyp presence in an image region. Brighter areas of these energy maps correspond to areas likely to contain a polyp inside. These energy maps are also used to guide the polyp segmentation.

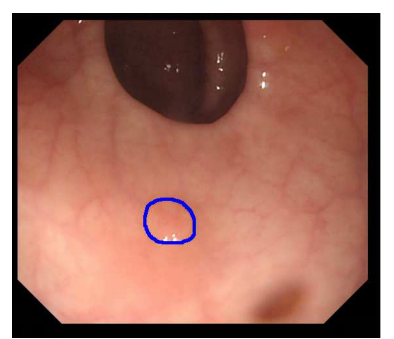

(a)

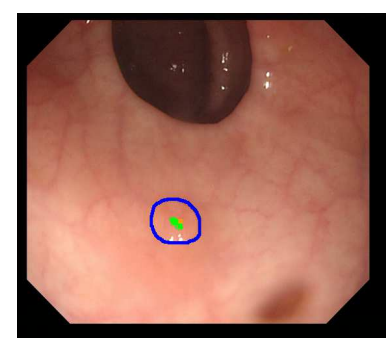

(b)

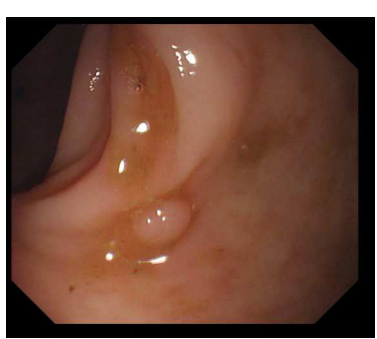

(c)

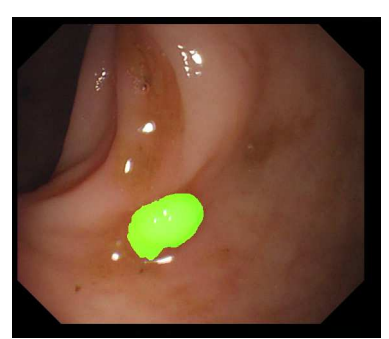

(d)

Figure 1: Examples of the outputs of the algorithms developed during the thesis: Polyp Localization (a-b) and Polyp Segmentation (c-d).

\footnotetext{
Correspondence to: $<$ jbernal@cvc.uab.es $>$
}

Recommended for acceptance by <Alicia Fornés and Volkmar Frinken $>$

ELCVIA ISSN:1577-5097

Published by Computer Vision Center / Universitat Autònoma de Barcelona, Barcelona, Spain 
Our methods achieve promising results in both polyp localization and segmentation -some examples are shown in Figure 1-. Our polyp localization method improves the performance of current state-of-the-art on the only public annotated database, which was also a contribution of this thesis. Our polyp segmentation method offers regions more accurate and richer in polyp content than state-of-the-art image segmentation methods. Finally, as we want to explore the practical applicability of our methods we present a comparative analysis between physicians fixations obtained via an eye tracking device and our polyp localization method. The results show that our method is indistinguishable to novice physicians although it is far from expert physicians.

\section{References}

[1] Jorge Bernal, F. Javier Sánchez, Fernando Vilariño "CURRENT CHALLENGES ON POLYP DETECTION IN COLONOSCOPY VIDEOS - From Region Segmentation to Region Classification. A Pattern Recognition-based Approach", Proceedings of the 2nd International Workshop on Medical Image Analysis and Description for Diagnosis Systems - MIAD 2011, pp. 62-71, Rome, Italy, January 2011

[2] Jorge Bernal, F. Javier Sánchez, Fernando Vilariño "A Region Segmentation Method for Colonoscopy Images Using a Model of Polyp Appearance", Proceedings of IbPRIA 2011, LNCS Vol. 6669, pp. 134143. Las Palmas de Gran Canaria, Spain, June 2011

[3] Jorge Bernal, Fernando Vilariño, F. Javier Sánchez "Towards Intelligent Systems for Colonscopy", In-Tech Colonoscopy Book, Chapter 16, pp. 245-270, July 2011.

[4] Jorge Bernal, F. Javier Sánchez, Fernando Vilariño. "Integration of Valley Orientation Distribution for Polyp Region Identification in Colonoscopy". In MICCAI 2011 Workshop on Computational and Clinical Applications in Abdominal Imaging (Vol. 6668, pp. 76-83), Lecture Notes in Computer Science, Springer Link, Toronto, Canada, September 2011

[5] Jorge Bernal, F. Javier Sánchez, Fernando Vilariño "Towards Automatic Polyp Detection With a Polyp Appearance Model”, Pattern Recognition (Vol. 45 Num. 9, pp. 3166-3182), 2012

[6] Joan Manel Nuñez, F. Javier Sánchez, Fernando Vilariño "Blood vessel characterization in colonoscopy images to improve polyp localization", In Proceeding of the 8th International Conference on Computer Vision Theory and Applications (Vol. 1, pp. 162-171), Barcelona, Spain, February 2013.

[7] Jorge Bernal, F. Javier Sánchez, Fernando Vilariño "Impact of Image Preprocessing Methods on Polyp Localization in Colonoscopy Frames", Proceedings of EMBC 2013, Osaka, Japan, July 2013

[8] Jorge Bernal, Fernando Vilariño, F. Javier Sánchez, M. Arnold, A. Ghosh, G. Lacey "Experts vs. Novices: Applying Eye-tracking Methodologies in Colonoscopy Video Screening for Polyp Search", Proceedings of ETRA 2014 Symposium on Eye Tracking Research and Applications, Florida, United States of America, March 2014 